\title{
Acute treatment with copoazú fermented extract ameliorates myocardial ischemia-reperfusion injury via eNOS activation
}

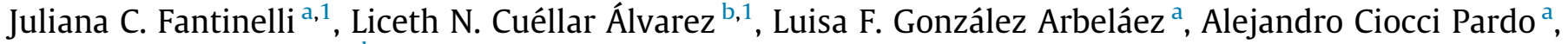 \\ Paula L. Galeano García ${ }^{b}$, Guillermo R. Schinella ${ }^{c}$, Susana M. Mosca ${ }^{a, *}$ \\ ${ }^{a}$ Centro de Investigaciones Cardiovasculares"Dr Horacio E. Cingolanï, CCT-CONICET, Universidad Nacional de La Plata, La Plata, Argentina \\ ${ }^{\mathrm{b}}$ Facultad de Ciencias Básicas, Universidad de la Amazonia, Florencia (Caquetá), Colombia \\ ' Cátedra de Farmacología Básica, Facultad de Ciencias Médicas, Universidad Nacional de La Plata, CIC, Provincia de Buenos Aires, La Plata, Argentina
}

\section{A R T I C L E I N F O}

\section{Article history:}

Received 16 February 2017

Received in revised form 11 April 2017

Accepted 3 May 2017

Available online 12 May 2017

\section{Keywords:}

Copoazú

Fermentation

Ischemia-reperfusion

NOS

Mitochondria

\begin{abstract}
A B S T R A C T
Our aim was to examine the effects of aqueous extracts of fermented (CFE) and non-fermented (CNFE) "copoazü (fruit of Theobroma grandiflorum) seeds against ischemia-reperfusion injury. Isolated rat hearts were submitted to $30 \mathrm{~min}$ of global ischemia (GI) and $60 \mathrm{~min}$ of reperfusion (R). Other hearts received CFE or CNFE in absence or in presence of L-NAME (a nitric oxide synthase inhibitor). Infarct size (IS) and postischemic myocardial function (PMF) were measured. Lipid peroxidation, reduced glutathione (GSH) and the expression of phosphorylated forms of eNOS, Akt, GSK-3 $\beta$ and PKC $\varepsilon$ were assessed. The response of isolated mitochondria to $\mathrm{Ca}^{2+}$ (MR) was also determined. CFE but not CNFE decreased IS, increased PMF and the expression of P-eNOS, P-Akt, P-GSK-3 $\beta$ and P-PKC $\varepsilon$, partially preserved GSH and improved MR. These effects were lost in presence of L-NAME.

These data demonstrate that the acute treatment with CFE protects the heart against ischemiareperfusion damage through NOS-dependent mechanisms.
\end{abstract}

(c) 2017 Elsevier Ltd. All rights reserved.

\section{Introduction}

Cardiovascular diseases and ischemic heart disease in particular, remain the leading cause of death and a significant cause of long-term morbidity worldwide. Although reperfusion is an absolute criterion for the survival of ischemic tissues, the notion has developed that it is not without hazard, and reperfusion-induced pathological changes may occur and further aggravate the previously ischemia-induced damage. An inadequate perfusion of myocardium despite removal of coronary artery occlusion, defined as no-reflow phenomenon̈, is one of the consequences to revascularization that causes left ventricular dysfunction, and even cardiac death (Chan et al., 2012).

Oxidative stress- imbalance of reactive oxygen species (ROS) production and antioxidants in favor of the former- and calcium overload appear as the most important contributors to the reperfusion injury (Das, 2001). Both factors impact on mitochondria promoting the formation and/or opening of mitochondrial

\footnotetext{
* Corresponding author at: Centro de Investigaciones Cardiovasculares "Dr Horacio E. Cingolanï, Universidad Nacional de La Plata, 60 y 120, 1900 La Plata, Argentina.

E-mail address: smosca@med.unlp.edu.ar (S.M. Mosca).

1 These authors collaborated equally to the work.
}

permeability transition pore (MPTP) leading to cell death (Halestrap, Clarke, \& Javadov, 2004).

The relationship between dietary factors and coronary heart disease has been and remains a matter of intense study and debate. Several studies show that an increase in fruit and vegetable consumption promotes cardiovascular health and reduces the ischemic stroke, the ischemic heart disease mortality and cardiovascular events (Gardener et al., 2011; Hlebowicz et al., 2013).

At experimental level, much attention has been focused on the beneficial actions against ischemia-reperfusion alterations achieved by plant extracts containing the polyphenols as predominant ingredients (Ma et al., 2013; Sakanashi et al., 2013; Saravanan, Ponmurugan, Sathiyavathi, Vadivukkarasi, \& Sengottuvelu, 2013). In this sense, we recently showed that the acute treatment with an extract of Teobroma cocoa (González Arbeláez et al., 2016) was able to limit the infarct size and MPTP opening through nitric oxide synthase (NOS) activation by reperfusion injury salvage kinases (RISK) pathways.

Theobroma grandiflorum is specie from the Amazon region that belongs to the Malvaceae family, as the cocoa tree (Theobroma cacao). The fruit pulp is used in the industry for candy, ice cream, liquor, and juice production while the seeds are used for the manufacturing of a product similar to chocolate called" cupulatë. 
Phytochemical studies show that the seeds and pulp of "copoazú contain flavone, flavan-3-ols and proanthocyanidin as principal polyphenols (Pugliese, Tomas-Barberan, Truchado, \& Genovese, 2013; Yang et al., 2003). There are scarce studies about "copoazú and these are mainly focused on the fruit pulp (Gonçalves, Lajolo, \& Genovese, 2010). Thus, it was previously demonstrated that daily administration of "copoazú liquors to diabetic rats ameliorated body weight gain, improved the plasma antioxidant capacity and reduced lipid peroxidation (Pinent et al., 2016). Recently, the antioxidant effects of a proanthocyanidin-rich extract from "copoazú " seeds in intestinal cells were shown (de Oliveira \& Genovese, 2013). However, the potential benefits against ischemia-reperfusion injury of the chocolate-like product obtained from "copoazú seeds have not yet been explored.

The present study was designed to (i) evaluate, in isolated and Langendorff perfused rat heart, the effects of "copoazú seeds aqueous extracts obtained at time 0 and after 10 days of traditional fermentation, against ischemia-reperfusion injury and (ii) identify the intracellular cascades responsible for the cardiac effects achieved by the extracts.

\section{Materials and methods}

\subsection{Plant material}

The fruit of Teobroma grandiflorum (copoazú), constitutes the material used for the fermentation in this research. Mature fruit were collected at the Center for Research of University of Amazonia CIMAZ-Macagual (voucher specimen number HUAZ 9030) located in the department of Caquetá, Colombia.

\subsection{Fermentation process}

The maceration was performed in the traditional fashion, punching 800 grams of "copoazú" seeds with $100 \%$ of pulp and the fermentation was carried out using baskets placed in the form of a ladder. The material was transferred from one basket to another and this removal was performed every $24 \mathrm{~h}$ after the start of the process for 10 days. After that the seeds were dried, toasted and crushed (Krysiak, 2006).

\subsection{Preparation of "copoazú" extracts}

$50 \mathrm{~g}$ of "copoazú" seeds obtained at 0 (CNFE) and 10 days (CFE) of fermentation process were solubilized in aqua and cooked for $20 \mathrm{~min}$. Both aqueous extracts were filtered, lyophilized, and maintained at $-30^{\circ} \mathrm{C}$ until used.

\subsection{HPLC analysis}

HPLC-DAD analysis was performed on a Shimadzu LC-2010HT system equipped with a pump LC-2010AHT, UV Detector LC2010AHT, autosampler LC2010 HT, and column RP-C18 $(150 \times 3 \mathrm{~mm}, 4 \mu \mathrm{m}$, Restek-Pinnacle). The data were collected and processed with the software LC- Solutions (Shimadzu). The experimental protocol was suggested by Hatzidimitriou et al. (2007) with some modifications. The eluting system used was: $90 \%$ solvent A (water with $2 \%$ acetic acid, v/v) and $10 \%$ solvent B (acetonitrile) in isocratic conditions. Peak identification was based on retention times and spiking with standard solutions. Sevenpoint calibration curves of theobromine, caffeine, $(+)$-catechin and (-)-epicatechin were used for quantification. The content of these compounds was expressed as $\mathrm{mg} / 100 \mathrm{~g}$ of dry weight of the extract.

\subsection{Determination of total phenols}

Total phenolic content was measured by using the FolinCiocalteu method. Results were expressed as mg gallic acid equivalents/100 g of dry weight of the extract (Singleton \& Rossi, 1965).

\subsection{Total antioxidant activity}

\subsubsection{Ferric reducing-antioxidant power (FRAP)}

The FRAP assay was carried out to determine the reducing ability of the extracts with a method adapted from Benzie and Strain (1996). Results were expressed as $\mu \mathrm{mol}$ of ascorbic acid equivalents/100 $\mathrm{g}$ of dry weight of the extract.

\subsubsection{1,1-Diphenyl-2-picryl-hydrazyl (DPPH) scavenging activity}

The reduction of DPPH stable free radical was determined according to the modified version of the method described by Cavin, Hostettmann, Dyatmyko, and Potterat (1998). Results are expressed in $\mu \mathrm{mol}$ Trolox/100 g of dry weight of the extract.

\subsection{Isolated heart preparation}

All procedures followed during this investigation were approved by the Institutional Animal Care and Use Committee (IACUC) of the Faculty of Medicine, University of La Plata (P-052014).

Hearts from male Wistar rats were isolated and Langendorff perfused with Ringer's solution containing (in mmol/L) $118 \mathrm{NaCl}$, $5.9 \mathrm{KCl}, 1.2 \mathrm{MgSO}_{4}, 1.35 \mathrm{CaCl}_{2}, 20 \mathrm{NaCO}_{3} \mathrm{H}$ and 11.0 glucose (gassed with $95 \% \mathrm{O}_{2}-5 \% \mathrm{CO}_{2}, \mathrm{pH} 7.4,37^{\circ} \mathrm{C}$ ). The conductive tissue in the atrial septum was damaged with a fine needle to achieve atrioventricular block, and the right ventricle was paced at $280 \pm 10$ beats/ min. A latex balloon tied to the end of a polyethylene tube was passed into the left ventricle through the mitral valve; the opposite end of the tube was then connected to a Statham P23XL pressure transducer. The balloon was filled with water to provide a left ventricular end-diastolic pressure (LVEDP) of $8-12 \mathrm{mmHg}$, and this volume was unchanged for the rest of the experiment. Coronary perfusion pressure (CPP) was monitored at the point of cannulation of the aorta and was adjusted to approximately 60-70 $\mathrm{mmHg}$. Coronary flow $(\mathrm{CF})$, which was controlled with a peristaltic pump, was $11 \pm 2 \mathrm{ml} / \mathrm{min}$. Left ventricular pressure (LVP) and CPP data were acquired by using an analog-to-digital converter and acquisition software (Chart V4.2.3 AD Instruments).

\subsubsection{Experimental protocols}

After 20 min of stabilization, the following experimental protocols were performed (Fig. 1):

Non-ischemic control hearts (NIC; $n=7$ ): Hearts were perfused for 110 min without any treatment.

Ischemic control hearts (IC, $n=8$ ): Hearts were subjected to $30 \mathrm{~min}$ of normothermic global ischemia followed by $60 \mathrm{~min}$ of reperfusion. Global ischemia was induced by stopping the perfusate inflow line, and the heart was placed in a saline bath held at $37^{\circ} \mathrm{C}$.

Copoazú non-fermented extract (CNFE $n=7$ ): Hearts were treated during the first $10 \mathrm{~min}$ of reperfusion with a dose of $0.30 \mathrm{mg} /$ min of CNFE.

Copoazú fermented extract (CFE $n=8)$ : Hearts were treated during the first $10 \mathrm{~min}$ of reperfusion with a dose of $0.30 \mathrm{mg} / \mathrm{min}$ of CFE. The final concentration in the perfusate was $30 \mu \mathrm{g} / \mathrm{ml}$.

L-NAME $(n=7)$ : Hearts received $1 \mathrm{mM}$ of $\mathrm{L}^{\mathrm{G}}$ nitro-L-arginine methyl ester (L-NAME) -a non-selective nitric oxide synthase (NOS) inhibitor- from $10 \mathrm{~min}$ before ischemia and during all the reperfusion time. 


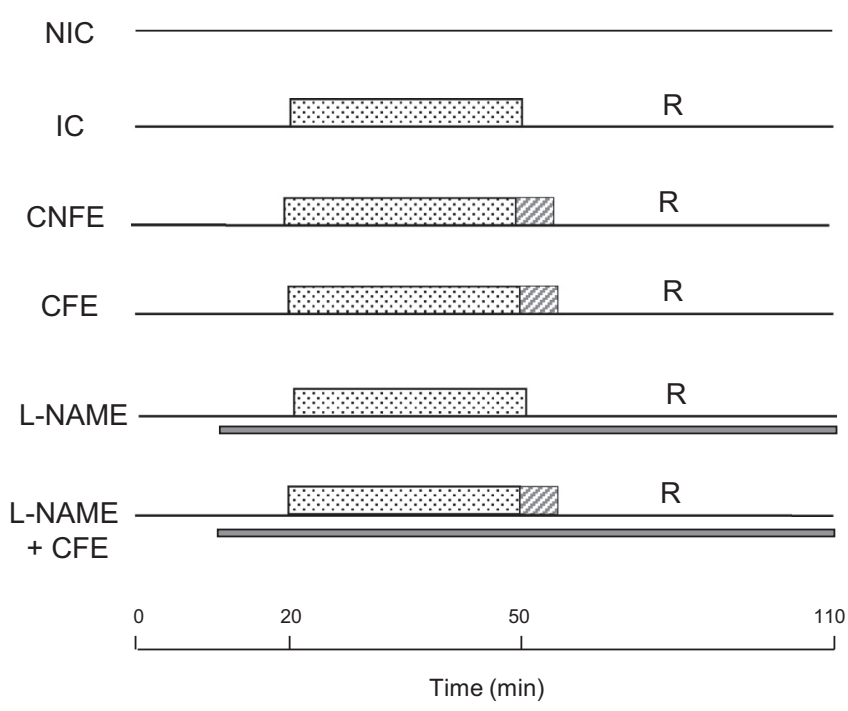

Fig. 1. Scheme of the experimental protocols. NIC: non-ischemic control; IC: ischemic control; CNFE: copoazú non-fermented extract; CFE: copoazú fermented extract; L-NAME: $\mathrm{L}^{\mathrm{G}}$-nitroL-arginine methyl ester, inhibitor of NOS and L-NAME $+\mathrm{CFE}$

L-NAME + CFE $(n=7)$ : Hearts received L-NAME in a similar manner than L-NAME group and $\mathrm{CP}$ was administered at the time and dose above indicated.

\subsubsection{Infarct size determination}

Infarct size was assessed by the widely validated triphenyltetrazolium chloride (TTC) staining technique (Fishbein et al., 1981). This technique relies on the ability of dehydrogenase enzymes and cofactors in the tissue to react with tetrazolium salt to form a formazan pigment (red). It is a recognized method to discriminate between dead and viable tissue. At the end of reperfusion, atrial and right ventricular tissues were excised and left ventricle (LV) was frozen. The freeze LV was cut into six transverse slices, which were incubated for $5 \mathrm{~min}$ at $37^{\circ} \mathrm{C}$ in a $1 \%$ solution of TTC. To measure myocardial infarction, the slices were weighed and scanned. The infarcted (pale) and viable ischemic/reperfused (red) areas were measured by computed planimetry (Scion Image 1.62; Scion Corp., Frederick, Maryland, USA). Infarct weights were calculated as $(\mathrm{A} 1 \times \mathrm{W} 1)+(\mathrm{A} 2 \times \mathrm{W} 2)+(\mathrm{A} 3 \times \mathrm{W} 3)+(\mathrm{A} 4 \times \mathrm{W} 4)$ $+(\mathrm{A} 5 \times \mathrm{W} 5)+(\mathrm{A} 6 \times \mathrm{W} 6)$, where $A$ is the area of infarct for the slice and $\mathrm{W}$ is the weight of the respective section. Infarct size was expressed as a percentage of total area (area at risk, AR).

\subsubsection{Systolic and diastolic function}

The systolic function was assessed by the left ventricular developed pressure (LVDP)- calculated by subtracting LVEDP from the left ventricular (LV) peak pressure values- and the maximal velocity of rise of LVP $\left(+\mathrm{dP} / \mathrm{dt}_{\max }\right)$. The diastolic function was evaluated through the maximal velocity of decrease of $\operatorname{LVP}\left(-\mathrm{dP} / \mathrm{dt}_{\max }\right)$ and LVEDP.

\subsubsection{Coronary resistance $(C R)$}

CR was calculated as a quotient between $\mathrm{CPP}$ and $\mathrm{CF}$.

\subsubsection{Assessment of lipid peroxidation}

We used the TBARS spectroscopic technique to evaluate lipid peroxidation. At the end of the reperfusion period, a portion of LV was homogenized in physiological saline solution and centrifuged at $770 \mathrm{~g}$ to allow measuring TBARS in the supernatant. Absorbance at $535 \mathrm{~nm}$ was measured and TBARS expressed in
Table 1

Content of phenolic compounds and antioxidant activity of aqueousextracts of copoazú.

\begin{tabular}{lll}
\hline & CNFE & CFE \\
\hline Phenolic compounds & & \\
Total phenols $(\mathrm{mg} \mathrm{GA} / 100 \mathrm{~g})$ & $1216 \pm 59$ & $189 \pm 3^{* *}$ \\
Catechin $(\mathrm{mg} / 100 \mathrm{~g})$ & $6.2 \pm 0.7$ & $3.6 \pm 0.4^{* *}$ \\
Epicatechin $(\mathrm{mg} / 100 \mathrm{~g})$ & $1.8 \pm 0.2$ & $1.0 \pm 0.1^{* *}$ \\
Theobromine $(\mathrm{mg} / 100 \mathrm{~g})$ & $2.7 \pm 0.3$ & $1.6 \pm 0.2^{* *}$ \\
Caffeine $(\mathrm{mg} / 100 \mathrm{~g})$ & $3.6 \pm 0.4$ & $2.1 \pm 0.2^{* *}$ \\
Antioxidant activity & & \\
FRAP $(\mu \mathrm{mol}$ AA/100 g) & $920 \pm 130$ & $130 \pm 2^{* *}$ \\
DPPH ( $\mu$ mol TROLOX/100 g) & $805 \pm 97$ & $144 \pm 3^{* *}$ \\
\hline
\end{tabular}

GA: Gallic acid; FRAP: Ferric reducing ability of plasma; AA: Ascorbic acid; DPPH: $\alpha$, $\alpha$-diphenyl- $\beta$-picrylhydrazyl. $\mathrm{p}<0.01$.

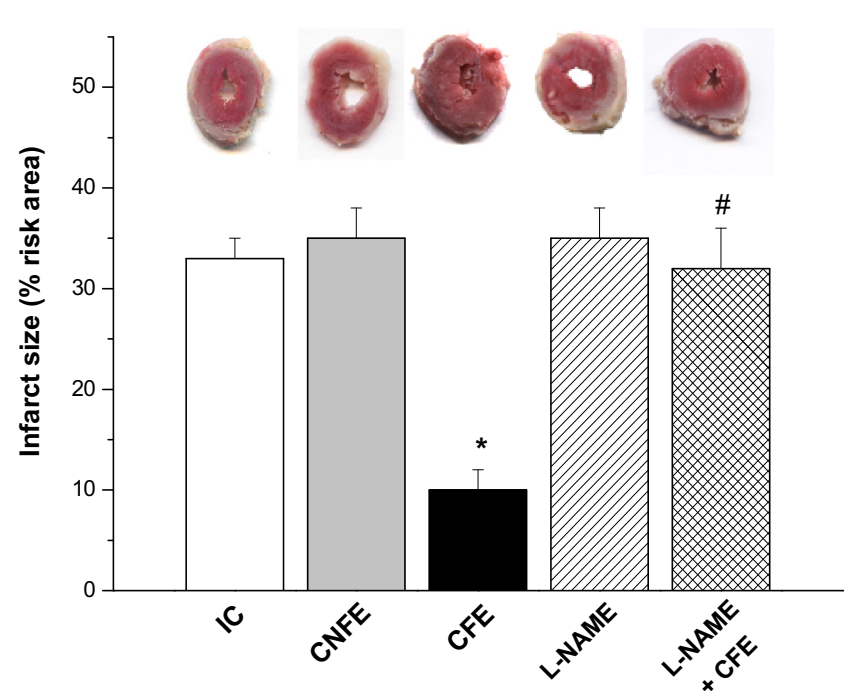

Fig. 2. Infarct size (IS), expressed as a percentage of risk area, in ischemic control (IC) and in hearts treated with aqueous extracts of copoazú fermented (CFE) and non-fermented (CFE) or L-NAME, inhibitor of NOS and the combination of L-NAME + CFE. Observe that CFE treatment but not CNFE decreased the IS obtained in IC hearts and that this action was abolished by L-NAME treatment. "p $<0.05$ vs. IC; ${ }^{\#} \mathrm{p}<0.05$ vs. CFE.

$\mathrm{nmol} / \mathrm{mg}$ protein using an extinction coefficient of $1.56 \times 105 \mathrm{M}^{-1} \mathrm{~cm}^{-1}$ (Buege \& Aust, 1974).

\subsubsection{Reduced glutathione}

GSH was determined by Ellman's method, which is based on the reaction of non-protein sulfhydryl groups with 5,5'-dithiobis (2nitrobenzoic acid) to give a compound that absorbs at $412 \mathrm{~nm}$. GSH levels were expressed as $\mu \mathrm{g} / \mathrm{mg}$ protein (Sedlak \& Lindsay, 1968).

\subsubsection{Immunoblotting}

Other portion of LV was homogenized and cytosolic fraction was isolated by differential centrifugation. Briefly, LV were homogenized in ice-cold RIPA buffer $(300 \mathrm{mmol} / \mathrm{L}$ sacarosa, $1 \mathrm{mmol} / \mathrm{L}$ DTT, $4 \mathrm{mmol} / \mathrm{L}$ EGTA, $20 \mathrm{mmol} / \mathrm{L}$ Tris $\mathrm{pH} 7.4$, 1\% Triton $\mathrm{X}, 10 \%$ protease cocktail, $25 \mu \mathrm{mol} / \mathrm{L} \mathrm{FNa}, 1 \mu \mathrm{mol} / \mathrm{L}$ ortovanadate) and centrifuged at $12,000 \mathrm{~g}$ for $15 \mathrm{~min}$ at $4{ }^{\circ} \mathrm{C}$. From supernatant proteins $(60 \mu \mathrm{g})$ were resolved on SDS-PAGE and transferred to PVDF membrane $(2 \mathrm{~h})$. Equal loading of samples was confirmed by Ponceau $\mathrm{S}$ staining. Membranes were blocked with $5 \%$ non-fat milk in Trisbuffered saline ( $\mathrm{pH} 7.5$ ) containing $0.1 \%$ Tween (TBS-T), and probed overnight at $4{ }^{\circ} \mathrm{C}$ with antibodies against phosphorylated 

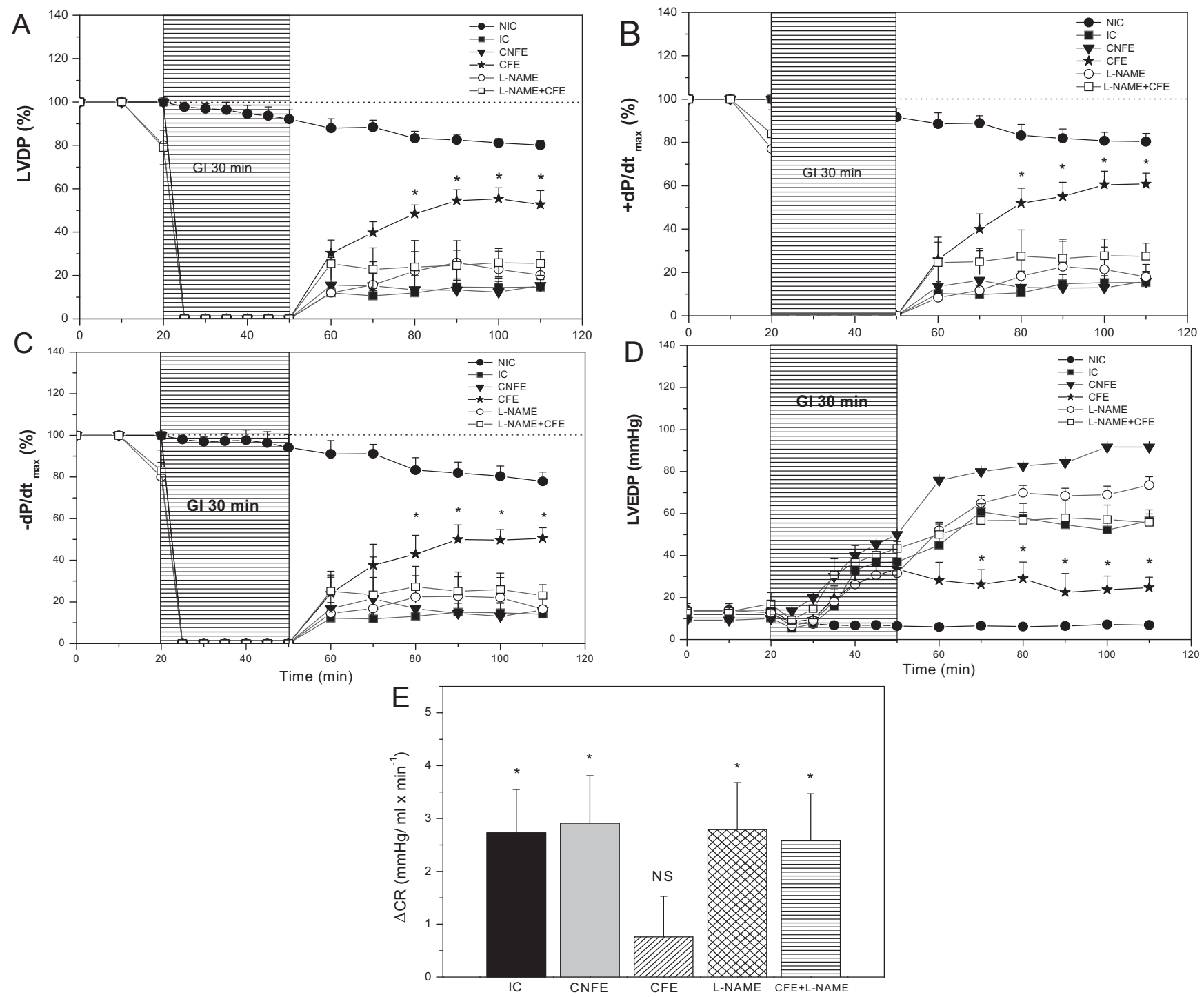

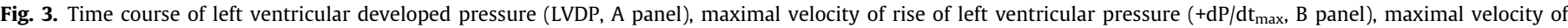

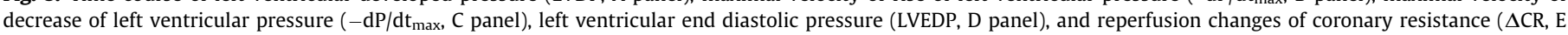

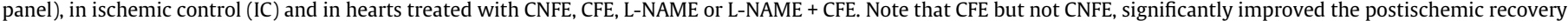

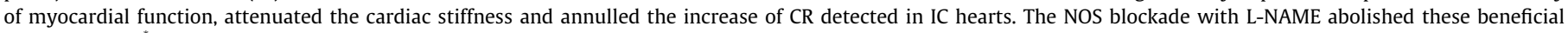
actions of CFE. " p $<0.05$ vs. IC.

forms of GSK-3 $\beta$-Ser9 (1:1000, Santa Cruz Biotechnology), Akt (1:1000, Santa Cruz Biotechnology), PKC $\varepsilon$ ((1:1000, Santa Cruz Biotechnology) and eNOS-Ser1177 (1:1000, Sigma-Aldrich). Membranes were washed four times for $10 \mathrm{~min}$ in TBS-T prior to addition of anti-rabbit secondary antibody (1:5000, Santa Cruz Biotechnology) and protein bands were analysed by a chemiluminescent system (ECL Plus; GE Healthcare Life Sciences). GAPDH signal was used as a loading control.

\subsection{Isolation of mitochondria}

LV of non-perfused rat hearts were washed and homogenized in ice-cold isolation solution (S) consisting of $75 \mathrm{mM}$ sucrose, $225 \mathrm{mM}$ mannitol, and $0.01 \mathrm{mM}$ EGTA neutralized with Trizma buffer at $\mathrm{pH}$ 7.4. After the tissue pieces were settled, the entire supernatant was discarded and fresh IS $(5 \mathrm{~mL})$ was added, and the mixture was transferred to a hand homogenizer. Proteinase ( $0.8 \mathrm{mg}$, bacterial, type XXIV, Sigma-Aldrich, formerly called Nagarse) was added just before starting the homogenization procedure. The whole homogenization procedure took no longer than $14 \mathrm{~min}$ in two steps of $7 \mathrm{~min}$ each (with $5 \mathrm{~mL}$ addition of fresh $\mathrm{S}$ each). The homogenate was carefully transferred after each step to a polycarbonate centrifuge tube. After $5 \mathrm{~min}$ of $480 \mathrm{~g}$ of centrifugation to discard unbroken tissue and debris, the supernatant was centrifuged at $7700 \mathrm{~g}$ for $10 \mathrm{~min}$ to sediment the mitochondria. The mitochondrial pellet was washed twice with IS and the last one with suspension solution (IS without EGTA) at $7700 \mathrm{~g}$ for $5 \mathrm{~min}$ each.

\subsection{1. $\mathrm{Ca}^{2+}$-induced mPTP opening}

The ability of mitochondria to resist swelling was assessed by incubating $0.3 \mathrm{mg} / \mathrm{mL}$ of isolated mitochondria in a buffer containing (in $\mathrm{mmol} / \mathrm{L}$ ): $120 \mathrm{KCl}, 20$ MOPS, 10 Tris $\mathrm{HCl}$, and $5 \mathrm{KH}_{2} \mathrm{PO}_{4}$ adjusted to $\mathrm{pH}=7.4$. After 5 -min preincubation, the mitochondria energized with the addition of $5 \mathrm{mmol} / \mathrm{L}$ succinate were induced to swell with $100 \mu \mathrm{mol} / \mathrm{L} \mathrm{CaCl}_{2}$. If $\mathrm{mPTP}$ is open in the presence of $\mathrm{Ca}^{2+}$ loading, solutes will be free to enter the inner matrix, causing the mitochondria to swell. These changes are observed as 

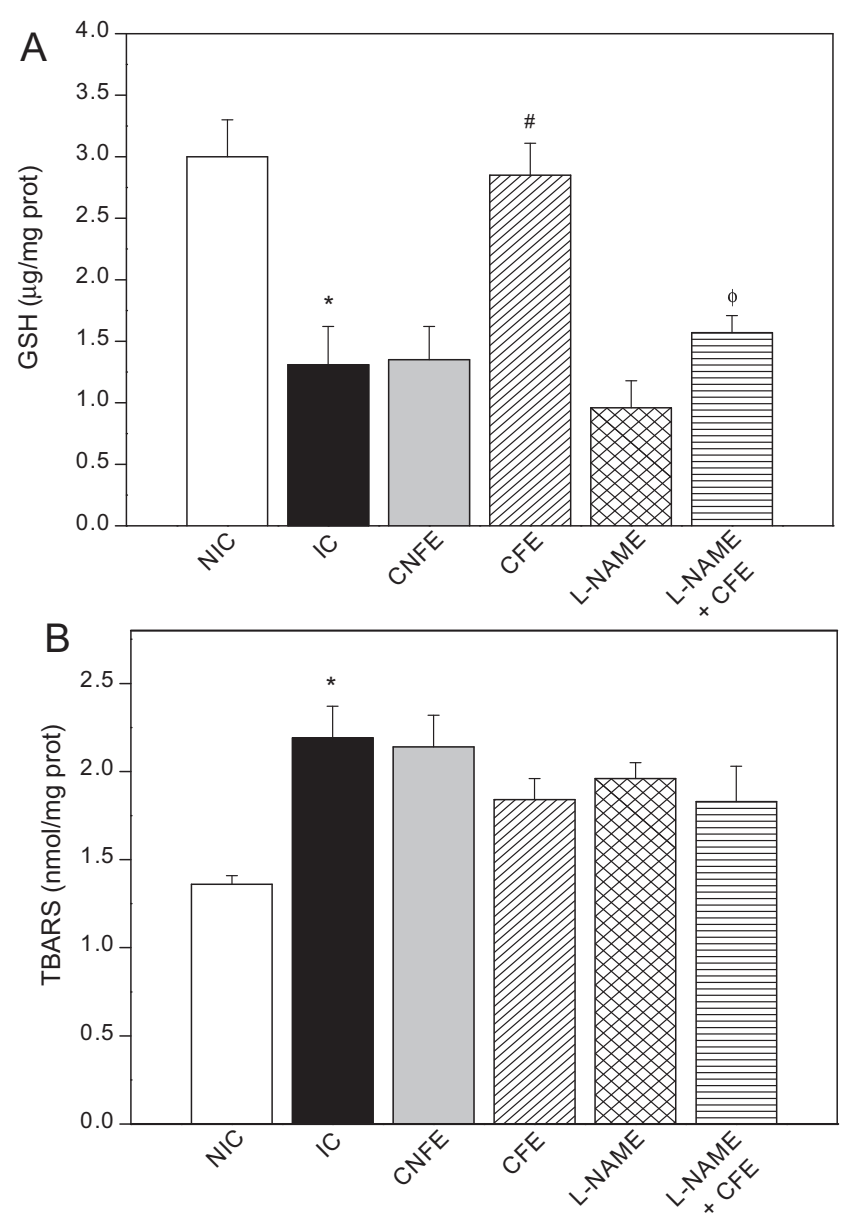

Fig. 4. Reduced glutathione content (GSH, A panel) and thiobarbituric acid reactive substances concentration (TBARS, B panel) in ischemic control hearts (IC) and in hearts treated with CNFE, CFE, L-NAME or L-NAME + CFE. GSH content decreased in IC hearts, CFE partially preserved it and L-NAME abolished the CFE action. The TBARS increased in IC hearts and were not modified by any treatment. " $\mathrm{p}<0.05$ vs. NIC; ${ }^{*} \mathrm{p}<0.05$ vs. IC; ${ }^{\phi} \mathrm{p}<0.05$ vs. CFE.

decreases of light scattering and followed using a temperaturecontrolled Hitachi F4500 spectrofluorometer operating with continuous stirring at excitation and emission wavelengths of 520 nm (Facundo, de Paula, \& Kowaltowski, 2007). LSD was calculated for each sample by taking the difference of scattered light between before and after the addition of $\mathrm{CaCl}_{2}$. LSD was assessed in samples without any treatment and in those treated with CFE $(7.5 \mu \mathrm{g} / \mathrm{mL})$, L-NAME $(1 \mathrm{mM})$ and the combination of both (LNAME + CFE). In order to relate mPTP opening to decreased light scattering, we added cyclosporine A $0.5 \mu \mathrm{M}$ to inhibit $\mathrm{MPTP}$ or abolish any observed reduction.

\subsection{Statistical analysis}

Data were expressed as means \pm SD. Statistical analysis was performed by one-way analysis of variance (ANOVA) followed by the Newman-Keul's post-test used for multiple comparisons among groups. Values of $\mathrm{p}<0.05$ were considered to indicate statistical significance.

\section{Results}

Table 1 shows the content of phenolic compounds and antioxidant activity of both "copoazú extracts (CNFE and CFE). The data show that CFE, probably as a consequence of the fermentation process, has a smaller amount of catechin, epicatechin, theobromine and caffeine and a lesser antioxidant activity than CNFE.

Thirty min of global ischemia followed by $60 \mathrm{~min}$ of reperfusion caused an infarct size (IS) of $\sim 30 \%$ of risk area. This value was not modified by the addition of CNFE or $\mathrm{L}^{\mathrm{G}}$-nitro-L-arginine methyl ester (L-NAME) but a significant reduction was observed when CFE $30 \mu \mathrm{g} / \mathrm{mL}$ was added to the perfusate (Fig. 2). This beneficial effect was annulled by L-NAME treatment.

The time course of contractility during ischemia and reperfusion of all experimental groups is shown in Fig. 3. In IC hearts, LVDP decreased to $15 \pm 4 \%$ from pre-ischemic value at the end of the reperfusion period. The treatment with CFE but not with CNFE improved post-ischemic recovery of myocardial function. At the end of reperfusion, LVDP reached a value of approximately 50\% of pre-ischemic value (Fig. 3, A panel). A similar pattern was observed when $+\mathrm{dP} / \mathrm{dt}_{\max }$ was analysed (Fig. 3, B panel). An increase of $-\mathrm{dP} / \mathrm{dt}_{\max }$ was also evident after CFE treatment (Fig. 3, C panel). The LVEDP (an index of diastolic stiffness) was approximately $10 \mathrm{mmHg}$ at the end of stabilization period and significantly increased during ischemia-reperfusion reaching a value of approximately $50 \mathrm{mmHg}$ at the end of reperfusion. After CNFE or L-NAME addition, LVEDP acquired higher values than those observed in IC. However, the treatment with CFE significantly reduced the LVEDP values detected in untreated hearts (Fig. 3, D panel). These beneficial effects were lost when NO synthesis was inhibited by L-NAME. Ischemic contracture was not modified by CFE but increased in presence of L-NAME.

No-reflow phenomenon, a disorder that interrupts the microcirculation during reperfusion, is also involved in reperfusion injury (Kloner, 2011). In our experimental preparation, at constant coronary flow (CF), changes of coronary perfusion pressure (CPP) produce changes in coronary resistance (CR). These changes are indicating the incidence of treatments on vasomotor tone. The ischemia-reperfusion produced an increase of CR leading to an inadequate coronary perfusion endangering myocardial cell life and promoting cell death. This increase was not observed after CFE treatment indicating than an improving of post-ischemic coronary perfusion occurred in presence of the extract. This beneficial action was abolished by NOS inhibition with L-NAME, highlighting the NO-mediated CFE vasodilator effect (Fig. 3, E panel).

Untreated hearts (IC) also showed a diminution of GSH level and an increase of TBARS. The treatment with CFE partially preserved the GSH content and did not modify the TBARS concentration. The beneficial action on GSH was attenuated when CFE was administered in presence of L-NAME (Fig. 4).

A significant increase of the expression of phosphorylated forms of Akt, GSK-3 $\beta$, e-NOS and PKC $\varepsilon$ at the end of reperfusion period was observed in hearts treated with CFE. This increase was significantly attenuated when CFE was added in presence of L-NAME (Fig. 5).

Fig. 6 shows typical traces of swelling experiments $(A)$ and the mean values of light scattering decrease (LSD, B) produced by the addition of $100 \mu \mathrm{mol} / \mathrm{L} \mathrm{Ca}^{2+}$ to samples of mitochondrial suspension obtained from untreated and treated hearts. The CFE treatment significantly decreased the LSD produced by $\mathrm{Ca}^{2+}$ $(0.26 \pm 0.10$ vs. $1.40 \pm 0.14$ a.u. $)$ and L-NAME abolished this change $(0.80 \pm 0.10$ a.u.). The addition of CsA significantly attenuated the LSD produced by $\mathrm{Ca}^{2+}(0.10 \pm 0.02$ a.u.) (data not shown).

\section{Discussion}

The present study demonstrates for the first time that the treatment at the onset of reperfusion with "copoazü fermented extract (CFE) limits the infarct size and improves postischemic recovery of 
A
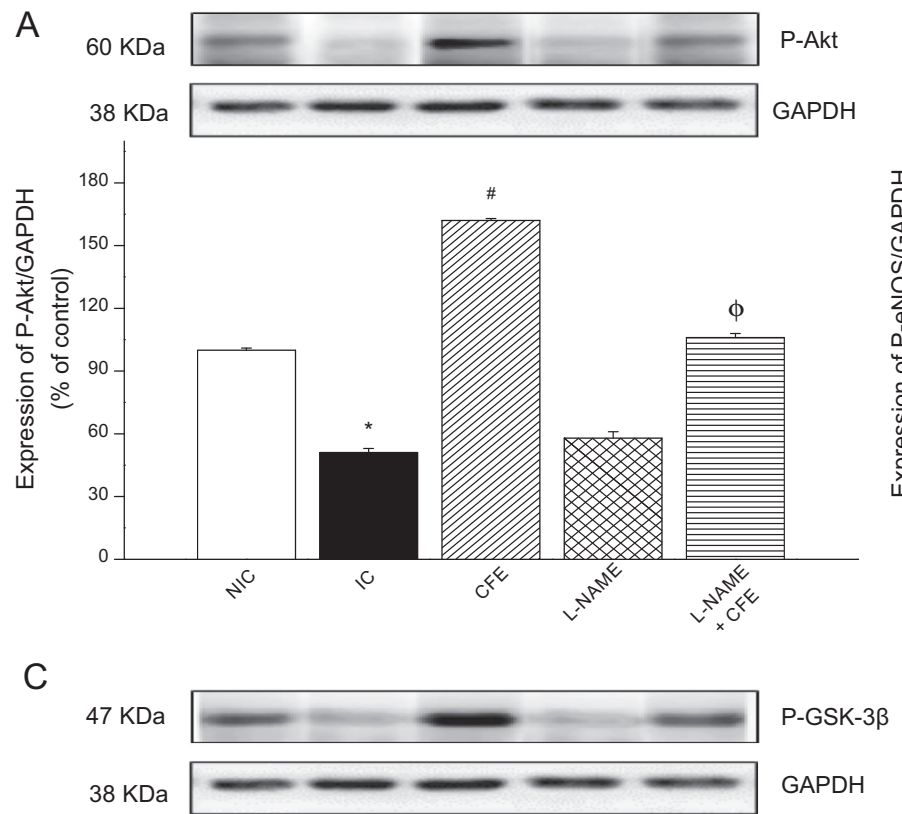

B
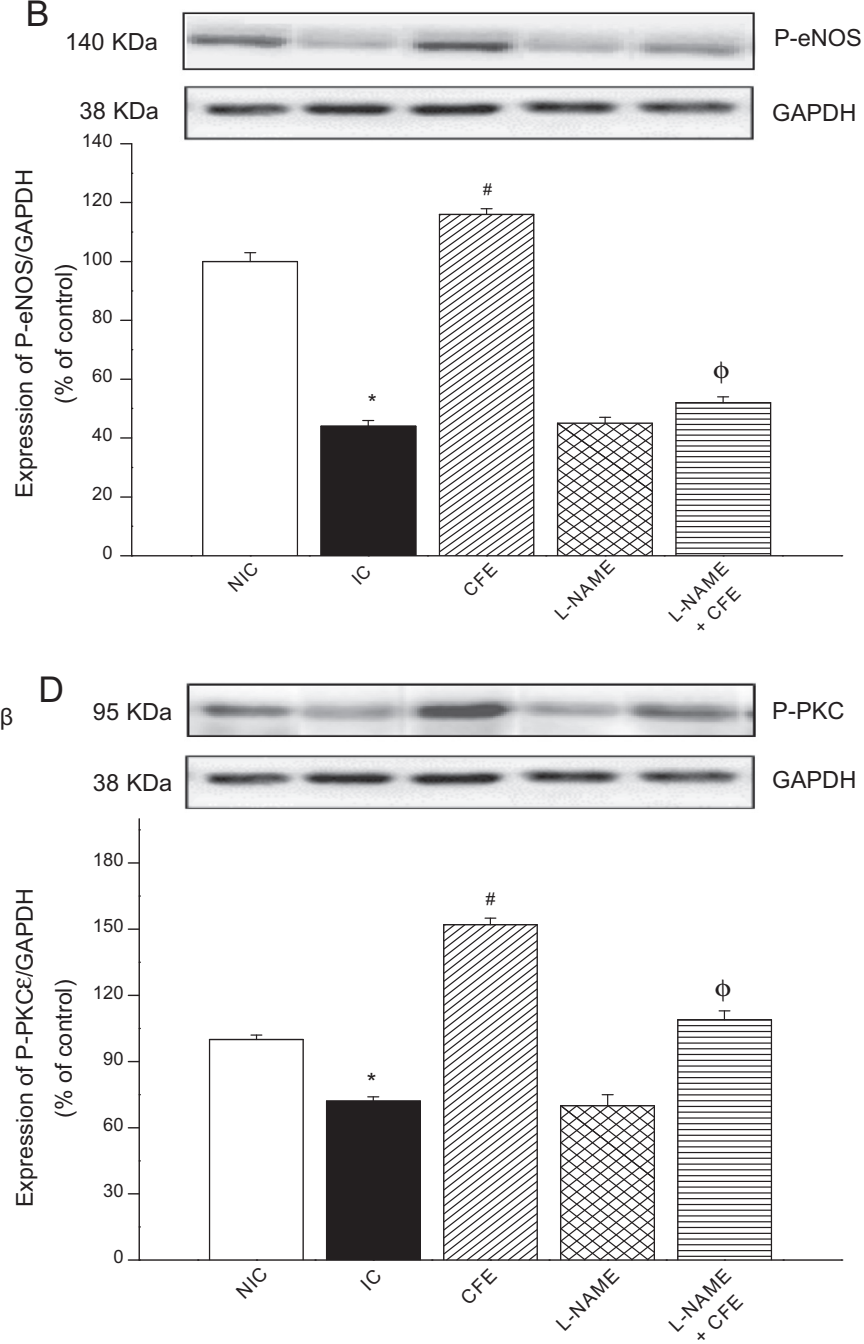

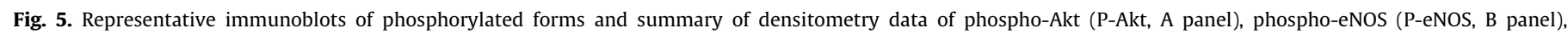

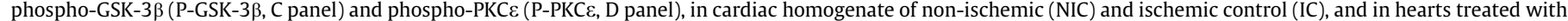

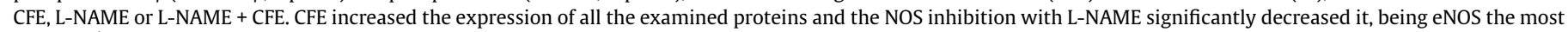
affected. ${ }^{*} \mathrm{p}<0.05$ vs. NIC; ${ }^{\#} \mathrm{p}<0.05$ vs. IC; ${ }^{\phi} \mathrm{p}<0.05$ vs. CFE.

myocardial function and coronary perfusion. In the same experimental conditions, the administration of a non-fermented extract of "copoazú did not exert beneficial actions on myocardium.

An improvement of coronary perfusion is an indication of the protection against "non-refloẅ phenomenon. Previous results suggest that polyphenols are able to improve endothelium-dependent vasodilation, in which NO plays a crucial role (Andriantsitohaina et al., 2012). In our experimental preparation, we detected an attenuation of coronary resistance (vasodilator effect) in hearts treated with CFE. This effect was abolished when NOS was inhibited by L-NAME. Therefore, this result reinforces the hypothesis that an increased NO production via eNOS activation achieved by CFE is an important mechanism contributing to the attenuation of coronary perfusion post-ischemic deficiency.

Of the various pathological events that lead to ischemiareperfusion injury, the abrupt ROS production, the increased cytosolic calcium and the cross talk between both events play key roles (Peng \& Jou, 2010). Although ROS production occurs in several cellular locations, the mitochondrial production predominates in ischemia-reperfusion-mediated oxidant injury in the heart. Under these conditions, the antioxidant effect should be relevant. In our experiments, the partial preservation of GSH detected in hearts treated with CFE would be an indication of a lesser ROS production. What would be the mechanism to explain this possible ROS diminution? Could it be due to the antioxidant action of the extract? There is accumulated evidence that show that cardioprotective mechanisms triggered by natural antioxidant go further than their antioxidant action (Akhlaghi \& Bandy, 2009). Examining our data this premise comes true. The" in vitrö analysis of CFE shows that the fermentation process, drying and roasting of " copoazú seeds, decreases the total polyphenols content and the antioxidant activity compared to CNFE. At this point it should be remembered that fermentation is an old technology used to improve the useful life and nutritional and organoleptic qualities of food and to extract its bioactive compounds (Martins et al., 2011). This process alters the relationship between nutritive and non-nutritive components of food, affecting the bioactivity and digestibility of the products (Zhang et al., 2012). In our case, cardioprotective actions against ischemia-reperfusion injury were only detected when CFE was administered. Therefore, we demonstrated that the product obtained by the fermentation process was efficient to protect the heart while that unfermented was not. Moreover, our results are suggesting that the antioxidant action of CFE is not the principal mechanism responsible of the 

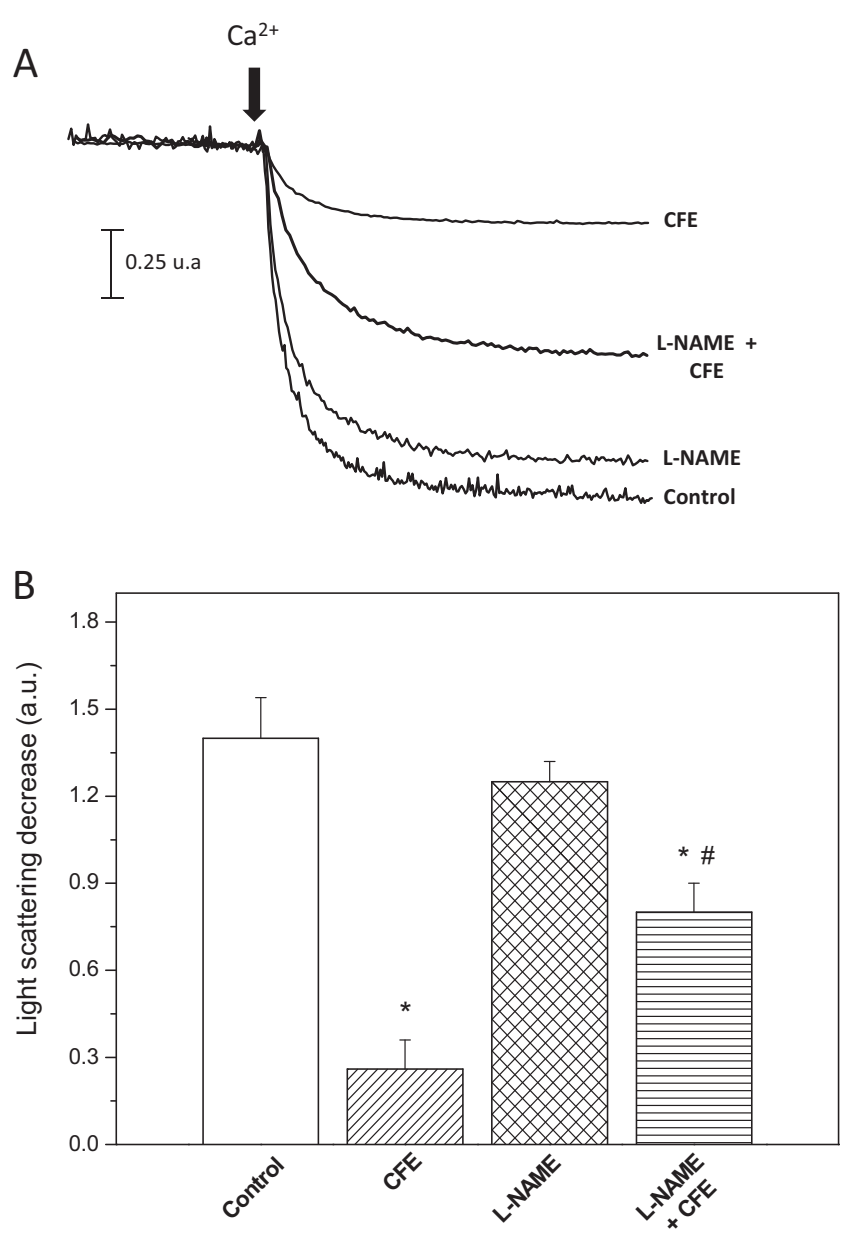

Fig. 6. Typical traces (A) and mean values of the light scattering decreases (B) produced by $100 \mu \mathrm{M} \mathrm{Ca}^{2+}$ addition to mitochondrial suspensions, in absence and in presence of "copoazú fermented extract (CFE), L-NAME or L-NAME + CFE. The response of isolated mitochondria to $\mathrm{Ca}^{2+}$ was significantly attenuated by CFE and partially restored after NOS inhibition with L-NAME. ${ }^{*} \mathrm{p}<0.05$ vs. C; ${ }^{\#} \mathrm{p}<0.05$ vs. CFE.

beneficial actions afforded by the extract against ischemiareperfusion injury. Hence, others mechanisms would be implicated. Thus, the modulation of intracellular signalling pathways has been long recognized as crucial for cardioprotection being the mitochondria and specifically the MPTP the end target. The formation/and opening of MPTP is linked to cell death (Halestrap et al., 2004) and the attenuation of that process promote the cell survival. In relation to the kinases, Juhaszova et al. (2009) showed that inhibition/phosphorylation of GSK-3 $\beta$ alters the phosphorylation of target substrates associated to MPTP thus delaying its opening time. Our data show that CFE increased the level of phosphorylated form of GSK-3 $\beta$ which was attenuated in presence of L-NAME.

What kinases are able to phosphorylate GSK-3 $\beta$ ? It was previously recognized that this action is mediated by PI3K/Akt dependent pathway (Tong, Imahashi, Steenbergen, \& Murphy, 2002). In this study the Akt expression followed the same pattern than GSK-3 $\beta$, i.e. decreased in IC, increased in CFE treated hearts and it was attenuated when NOS was inhibited.

On the other hand, it has been previously documented the protective role of NO during ischemia-reperfusion (Bolli, 2001). This radical combines with $\mathrm{O}_{2}^{-}$. at a very fast rate to form peroxynitrite $\left(\mathrm{ONOO}^{-}\right)$. NO is produced by nitric oxide synthase (NOS) activated by phosphorylation. Although there are many potential phospho- rylation sites on the endothelial isoform of NOS (eNOS), functionally most is known about the phosphorylation of the Ser1177 residue (induces eNOS activation) and the Thr495 residue (produces eNOS inhibition). In our experimental conditions, an increase of eNOS ${ }^{\text {Ser1117 }}$ phosphorylation was detected in CFE treated compared to untreated hearts and was annulled in presence of LNAME. Who activates eNOS? Previously, it was shown that Akt is able to activate eNOS increasing NO production (Fulton et al., 1999). Other kinases and phosphatases, such as PKC, ERK1/2, protein phosphatase 1 and protein phosphatase $2 \mathrm{~A}$ participate in the control of eNOS phosphorylation (Dudzinski \& Michel, 2007).

Protein kinase $C \varepsilon(P K C \varepsilon)$ is a pivotal signalling element in the myocardial protection against ischemia-reperfusion injury (Baines, Pass, \& Ping, 2001). An essential feature of the activation of PKC $\varepsilon$ is its subcellular redistribution. The translocation to specific subcellular compartments is thought to be an important mechanism for $\mathrm{PKC} \varepsilon$ to direct downstream signalling cascades and orchestrate protection. In this study, we showed an increase of the phosphorylated form of PKC $\varepsilon$ in hearts treated with CFE and a reduction in presence of L-NAME.

The decreased level of Akt and PKCE in presence of L-NAME could be explained considering the ability of NO/cGMP in the translocation/activation of PKC (Costa et al., 2005; Ping et al., 1999) and Akt phosphorylation (Kawasaki et al., 2003). A physical and functional coupling of PKC $\varepsilon$, Akt, and eNOS constituting signalling modules, as was previously reported (Zhang et al., 2005) could be contributing to the cardiac protection afforded by CFE.

Mitochondria and specifically the MPTP has received considerable attention in the development of cardioprotection. In isolated mitochondria we observed an attenuation of $\mathrm{MPTP}$ response to $\mathrm{Ca}^{2+}$ after CFE addition. This response was abolished in presence of L-NAME suggesting that NO plays a crucial role in the protective effects of CFE on mitochondria. This action could explain the CFEevoked infarct size limitation.

Given the complex composition of herbal extracts, the beneficial actions observed in hearts treated with CFE would be the result of the synergistic action of all components and not attributable only to polyphenols (Wagner \& Ulrich-Merzenich, 2009; Yang et al., 2014).

\section{Conclusion}

This study demonstrate in an"ex vivö heart model that the treatment only during reperfusion with an aqueous extract of fermented " copoazü seeds protects the myocardium against ischemia-reperfusion injury through Akt/GSK-3 $\beta$, Akt/NO/Akt, Akt/NO/PKCE-dependent pathways- in which NO plays a central role- targeting on mitochondria. Other interesting finding is that there is no direct relationship between antioxidant effect and cardioprotection. Taking into account the growing interest in designing food-derived tools for health improvement and for preventing and treating several pathologies, the knowledge of the beneficial effects of "copoazú fermented extract may be of great relevance. However, our findings could not be extrapolled directly to human. Multiple models including large animals and models with co-morbidities should be performed prior to clinical translation.

Since that the combined therapy is used to improve the patient's overall health status, the utilization of herbal medicine, which is considered as a "multi-target herb" should be optimized.

\section{Conflict of interest}

None. 


\section{Acknowledgment}

This study was supported by the Grant M-169 from the National University of La Plata of Argentina to Dr. S. Mosca.

\section{References}

Akhlaghi, M., \& Bandy, B. (2009). Mechanisms of flavonoid protection against myocardial ischemia-reperfusion injury. Journal of Molecular and Cellular Cardiology, 46, 309-317.

Andriantsitohaina, R., Auger, C., Chataigneau, T., Étienne-Selloum, N., Li, H. Martínez, M. C., ... Laher, I. (2012). Molecular mechanisms of the cardiovascular protective effects of polyphenols. British Journal of Nutrition, 108(9), 1532-1549.

Baines, C. P., Pass, J. M., \& Ping, P. (2001). Protein kinases and kinase-modulated effectors in the late phase of ischemic preconditioning. Basic Research in Cardiology, 96, 207-218.

Benzie, I. F. F., \& Strain, J. J. (1996). The ferric reducing ability of plasma (FRAP) as a measure of "anti-oxidant power": The FRAP assay. Analytical Biochemistry, 239 (1), 7076.

Bolli, R. (2001). Cardioprotective function of inducible nitric oxide synthase and role of nitric oxide in myocardial ischemia and preconditioning: An overview of a decade of research. Journal of Molecular and Cellular Cardiology, 33(11), 1897-1918.

Buege, J. A., \& Aust, S. D. (1974). Microsomal lipid peroxidation. Methods Enzymology, 52, 302-309.

Cavin, A., Hostettmann, K., Dyatmyko, W., \& Potterat, O. (1998). Anti-oxidant and lipophilic constituents of Tinospora crispa. Planta Medica, 64(5), 393396.

Chan, W., Stub, D., Clark, D. J., Ajani, A. E., Andrianopoulos, N., Brennan, A. L., Melbourne Interventional Group Investigators (2012). Usefulness of transient and persistent no reflow to predict adverse clinical outcomes following percutaneous coronary intervention. American Journal of Cardiology, 109, 478-485.

Costa, A. D., Garlid, K. D., West, I. C., Lincoln, T. M., Downey, J. M., Cohen, M. V., \& Critz, S. D. (2005). Protein kinase G transmits the cardioprotective signal from cytosol to mitochondria. Circulation Research, 97, 329-336.

Das, D. K. (2001). Redox regulation of cardiomyocyte survival and death. Antioxidants E Redox Signaling, 1, 23-37.

de Oliveira, T. B., \& Genovese, M. I. (2013). Chemical composition of Cupuassu (Theobroma grandiflorum) and Cocoa (Theobroma cacao) liquors and their effects on streptozotocin-induced diabetic rats. Food Reserch International, 51(2), 929-935.

Dudzinski, D. M., \& Michel, T. (2007). Life history of eNOS: Partners and pathways. Cardiovascular Research, 75, 247-260.

Facundo, H. T., de Paula, J. G., \& Kowaltowski, A. J. (2007). Mitochondrial ATPsensitive $\mathrm{K}+$ channels are redox-sensitive pathways that control reactive oxygen species production. Free Radical Biology E' Medicine, 42, 1039-1048.

Fishbein, M. C., Meerbaum, S., Rit, J., Lando, U., Kanmatsuse, K., Mercier, J. C., ... Ganz, W. (1981). Early phase acute myocardial infarct size quantification: Validation of the triphenyl tetrazolium chloride tissue enzyme staining technique. American Heart Journal, 101, 593-600.

Fulton, D., Gratton, J. P., McCabe, T. J., Fontana, J., Fujio, Y., Walsh, K., ... Sessa, W. C. (1999). Regulation of endothelium-derived nitric oxide production by the protein kinase Akt. Nature, 1999(99), 597-601.

Gardener, H., Wright, C. B., Gu, Y., Demmer, R. T., Boden-Albala, B., Elkind, M. S., ... Scarmeas, N. (2011). Mediterranean-style diet and risk of ischemic stroke, myocardial infarction, and vascular death: The Northern Manhattan Study. The American Journal of Clinical Nutrition, 94(6), 1458-1464.

Gonçalves, A. E., Lajolo, F. M., \& Genovese, M. I. (2010). Chemical composition and antioxidant/antidiabetic potential of Brazilian native fruits and commercial frozen pulps. Journal of Agricultural and Food Chemistry, 58(8), 4666-4674.

González Arbeláez, L. F., Ciocci Pardo, A., Fantinelli, J. C., Caldiz, C., Río, J. L., Schinella, G. R., \& Mosca, S. M. (2016). Ex vivo treatment with a polyphenol-enriched cocoa extract ameliorates myocardial infarct and postischemic mitochondrial injury in normotensive and hypertensive rats. Journal of Agricultural and Food Chemistry, 64(25), 5180-5187.

Halestrap, A. P., Clarke, S. J., \& Javadov, S. A. (2004). Mitochondrial permeability transition pore opening during myocardial reperfusion - a target for cardioprotection. Cardiovascular Research, 61, 372-385.
Hatzidimitriou, E., Nenadis, N., \& Tsimidou, M. Z. (2007). Changes in the catechin and epicatechin content of grape seeds on storage under different water activity (aw) conditions. Food Chemistry, 105(4), 1504-1511.

Hlebowicz, J., Drake, I., Gullberg, B., Sonestedt, E., Wallström, P., Persson, M., ... Wirfält, E. (2013). A high diet quality is associated with lower incidence of cardiovascular events in the Malmö diet and cancer cohort. PLoS ONE, 8(8), e71095.

Juhaszova, M., Zorov, D. B., Yaniv, Y., Nuss, H. B., Wang, S., \& Sollott, S. J. (2009). Role of glycogen synthase kinase-3 beta in cardioprotection. Cirulation Research, 104, 1240-1252.

Kawasaki, K., Smith, R. S., Jr., Hsieh, C. M., Sun, J., Chao, J., \& Liao, J. K. (2003). Activation of the phosphatidylinositol 3-kinase/protein kinase Akt pathway mediates nitric oxide-induced endothelial cell migration and angiogenesis. Molecular and Cellular Biology, 23(16), 5726-5737.

Kloner, R. A. (2011). No-Reflow phenomenon: Maintaining vascular integrity. Journal of Cardiovascular Pharmacology and Therapeutics, 16, 244-250.

Krysiak, W. (2006). Influence of roasting conditions on coloration of roasted cocoa beans. Journal of Food Engineering, 77, 449-453.

Ma, X., Zhang, K., Li, H., Han, S., Ma, Z., \& Tu, P. (2013). Extracts from Astragalus membranaceus limit myocardial cell death and improve cardiac function in a rat model of myocardial ischemia. Journal of Ethnopharmacology, 149(3), 720-728.

Martins, S., Mussatto, S. I., Martínez-Avila, G., Montañez-Saenz, J., Aguilar, C. N., \& Teixeira, J. A. (2011). Bioactive phenolic compounds: Production and extraction by solid-state fermentation. Biotechnology Advances, 29(3), 365-373.

Peng, T. I., \& Jou, M. J. (2010). Oxidative stress caused by mitochondrial calcium overload. Annals of the New York Academy Sciences, 1201, 183-188.

Pinent, M., Castell-Auví, A., Genovese, M. I., Serrano, J., Casanova, A., Blay, M., \& Ardévol, A. (2016). Antioxidant effects of proanthocyanidin-rich natural extracts from grape seed and cupuassu on gastrointestinal mucosa. Journal of the Science of Food and Agriculture, 96(1), 178-182.

Ping, P., Takano, H., Zhang, J., Tang, X. L., Qiu, Y., Li, R. C., ... Bolli, R. (1999). Isoformselective activation of protein kinase $C$ by nitric oxide in the heart of conscious rabbits: A signaling mechanism for both nitric oxide-induced and ischemiainduced preconditioning. Circulation Research, 84, 587-604.

Pugliese, A. G., Tomas-Barberan, F. A., Truchado, P., \& Genovese, M. I. (2013). Flavonoids, proanthocyanidins, Vitamin C, and antioxidant activity of Theobroma grandiflorum (Cupuassu) pulp and seeds. Journal of Agricultural and Food Chemistry, 61(11), 2720-2728.

Sakanashi, M., Matsuzaki, T., Noguchi, K., Nakasone, J., Sakanashi, M., Uchida, T., ... Tsutsui, M. (2013). Long-term treatment with san'o-shashin-to, a kampo medicine, markedly ameliorates cardiac ischemia-reperfusion injury in ovariectomized rats via the redox-dependent mechanism. Circulation Journal, 77(7), 1827-1837.

Saravanan, G., Ponmurugan, P., Sathiyavathi, M., Vadivukkarasi, S., \& Sengottuvelu, S. (2013). Cardioprotective activity of Amaranthus viridis Linn: Effect on serum marker enzymes, cardiac troponin and antioxidant system in experimental myocardial infarcted rats. International Journal of Cardiology, 165(3), 494-498.

Sedlak, J., \& Lindsay, R. H. (1968). Estimation of total, protein-bound, and nonprotein sulfhydryl groups in tissue with Ellman's reagent. Analytical Biochemistry, 25, 192-205.

Singleton, V. I., \& Rossi, J. A. Jr, (1965). Colorimetry of total phenolics with phosphomolybdic acid reagents. American Journal of Enology and Viticulture, 16 (3), 144158 .

Tong, H., Imahashi, K., Steenbergen, C., \& Murphy, E. (2002). Phosphorylation of glycogen synthase kinase-3beta during preconditioning through a phosphatidylinositol-3-kinase-dependent pathway is cardioprotective. Cirulation Research, 90(4), 377-379.

Wagner, H., \& Ulrich-Merzenich, G. (2009). Synergy research: Approaching a new generation of phytopharmaceuticals. Phytomedicine, 16, 97-110.

Yang, H., Protiva, P., Cui, B., Ma, C., Baggett, S., Hequet, V., ... Kennelly, E. J. (2003). New bioactive polyphenols from Theobroma grandiflorum ("cupuaçu"). Journal of Natural Products, 66, 1501-1504.

Yang, Y., Zhang, Z., Li, S., Ye, X., Li, X., \& He, K. (2014). Synergy effects of herb extracts: Pharmacokinetics and pharmacodynamic basis. Fitoterapia, 92 133-147.

Zhang, J., Baines, C. P., Zong, C., Cardwell, E. M., Wang, G., Vondriska, T. M., \& Ping, P. (2005). Functional proteomic analysis of a three-tier PKCepsilon-Akt-eNOS signaling module in cardiac protection. American Journal Physiology. Heart and Circulatory Physiology, 288(2), H954-H961.

Zhang, Z., Lv, G., Pan, H., Fan, L., Soccol, C. R., \& Pandey, A. (2012). Production of powerful antioxidant supplements via solid-state fermentation of wheat (Triticum aestivum Linn.) by cordyceps militaris. Food Technology and Biotechnology, 50(1), 32-39. 